\title{
Control of Spectral Shift of Plasmon Resonance of Ag-PVP Nanocomposite By Varying The PVP Shell Thickness In Core-Shell Structure
}

\section{Dilip Sao}

Sidho-Kanho-Birsha University

\section{Probodh Kumar Kuiri}

Sidho-Kanho-Birsha University

Rajib Nath ( $\nabla$ rajibnath.bu@gmail.com )

Sidho-Kanho-Birsha University https://orcid.org/0000-0002-3781-6906

\section{Research Article}

Keywords: Ag-PVP core-shell, Nanoparticles, Surface plasmon resonance, Blueshift, Shell thickness

Posted Date: July 23rd, 2021

DOl: https://doi.org/10.21203/rs.3.rs-689632/v1

License: (c) This work is licensed under a Creative Commons Attribution 4.0 International License.

Read Full License 


\title{
Control of spectral shift of plasmon resonance of Ag-PVP nanocomposite by varying the PVP shell thickness in core-shell structure
}

\author{
Dilip Sao · Subhamay Pramanik . \\ Probodh K. Kuiri · Rajib Nath*
}

July 5,2021

\begin{abstract}
Metal-polymer nanocomposite materials have interesting physical, chemical, and optical properties which were highly utilized in electronic, optical, and biomedical applications. Here, we have experimentally and theoretically studied the crucial role of polymer Polyvinylpyrrolidone (PVP) on the localized surface plasmon resonance (LSPR) spectra of silver (Ag) nanoparticles (NPs). In this work, we have synthesized PVP stabilized Ag NPs in Ethelene Glycol (EG) medium and observed a large blueshift $(\sim 20 \mathrm{~nm})$ of LSPR peak and tunable plasmonic properties of PVP coated Ag NPs by only varying the concentration of PVP. A reduction of Ag NP size from $\sim 13 \mathrm{~nm}$ to $\sim 5 \mathrm{~nm}$ with the increase in PVP concentration from $0.125 \mathrm{mM}$ to $3 \mathrm{mM}$ is seen. We found that the observed large blueshift of the LSPR bands with the varying in PVP concentration is not just due to the reduced Ag NP size but due to the combined effect of thickness of the PVP shell and radius of the Ag NP core. The polymer PVP used in the synthesis process of Ag NPs plays role of a surface modifier and forms a protective shell/layer surrounding the (core) particle. This is supported by the theoretical optical absorption data obtained from a modified effective medium theory. To accommodate such a large blueshift of the LSPR bands we have considered the Ag-PVP core-shell structure. The LSPR peak shift in the core-shell nanostructure is well accommodated by varying the effective shell thickness of PVP while taking the core size of Ag NPs as measured experimentally for each case. This clearly indicates that the observed large blueshift of SPR band of Ag NPs cannot be justified

Dilip Sao

Ramkrishna Mahato Government Engineering College, Purulia, West Bengal, India E-mail: dilipsaobbc@gmail.com

Subhamay Pramanik, Probodh K. Kuiri

Department of Physics, Sidho-Kanho-Birsha University, Purulia, West Bengal, India.

Rajib Nath* (* Corresponding Author)

* Department of Physics, Sidho-Kanho-Birsha University, Purulia, West Bengal, India. Email: *rajibnath.bu@gmail.com
\end{abstract}


by considering the decrease in Ag NP size alone. It is also confirmed that the shell thickness of PVP plays a crucial role in explaining the blueshift of the SPR band of Ag NPs. This study can help to understand the optical response especially plasmonic properties of other metal NPs coated with organic polymers.

Keywords Ag-PVP core-shell - Nanoparticles · Surface plasmon resonance · Blueshift · Shell thickness

\section{Introduction}

Silver nanoparticles (NPs) have been investigated for a long time due to their unique functional and optical properties which have been efficiently used in photo-catalytic [1,2], sensing [3,4, electronic [5, optical 6, 7] and bio-medical applications 8, 9, 10 . Among the various useful properties of Ag NPs, optical properties of these materials received huge attention from the scientific community due to the large tunable optical response in the visible to the near infrared region of the electromagnetic spectrum [11,12. The unique optical response or the optical absorption (OA) spectra of the noble metal NPs like Ag is due to the localized collective oscillations of the free surface electrons when resonates with the excitation of the external electromagnetic waves which is well known as localized surface plasmon resonance (LSPR) 13, 14. The LSPR effect in metal NPs enhances the electric field near the close vicinity of the metal NPs. This surface effect of metal NPs made them a useful materials for optical, chemical, and bio-material sensors [15,16,17]. The LSPR of the metal NPs was generally found to be dependent on particle size, shape, surrounding dielectric medium (solvent), and surface functionalization [18,19,20,21. Thus the OA spectra of metal NPs can be varied by changing these parameters. In this context, polymer-metal nanocomposite is a useful system to study the optical response of metal NPs as the polymer not only stabilized the NPs by preventing the agglomeration in the solvent medium but also modified the surface of the NPs by making a protective layer or shell surrounding it. Most of the previous experimental works which studied the variation in OA spectra of polymer (surfactant) stabilized metal NPs emphasized on the change in the particle size and shape when the solvent /dielectric medium is fixed but the role of polymer (surfactant) layer (or shell) was not well studied. These polymers can effectively change the dielectric behavior of the medium in close vicinity of the NPs 22]. This is the basis for the use of noble metal NPs as sensitive sensors. Therefore, it is important to study the effects of polymers as capping agents on the resonant absorption of the NPs.

In this work, we have experimentally and theoretically investigated the crucial role of polymer Polyvinylpyrrolidone (PVP) as a stabilizing agent on the OA spectra of Ag NPs synthesized in Ethylene Glycol (EG) medium. With the varying concentrations ( 0.125 to $3 \mathrm{mM}$ ) of PVP, we observed a large blueshift of $20 \mathrm{~nm}$ in the LSPR peak in the OA spectra of Ag NPs. To find out the role of the PVP layer in the observed large blueshift of the LSPR 
peak, we considered the Ag-PVP system as a core-shell structure where Ag $\mathrm{NP}$ is the metal core and the PVP layer is the protective shell. We performed theoretical calculations using the effective medium theory to obtain OA spectra for these core-shell NPs for each concentration of PVP using the uncoated Ag NP particle size (as estimated from the experimental data). From the theoretical calculations, we have shown that the decrease in Ag NP size can produce a blueshift of the LSPR peak but this shift is not comparable with the blueshift observed in the experimental OA data and also showed that the ratio of the core $(\mathrm{Ag} \mathrm{NP})$ to shell $(\mathrm{PVP})$ radius causes the observed large blueshift. We also found that the LSPR peak shift is actually linear with respect to the combined radius of the core and shell. Thus, we have provided both experimental and theoretical understanding of how the size of the NP core and the thickness of the polymer shell can simultaneously change the LSPR spectra of Ag NPs which may help to understand the optical response of other plasmonic NPs coated with polymer layers.

\section{Experiments}

Ag-NPs were synthesized by polyol process. All the chemicals were of analytical grade and used as purchased without any purification. We have synthesized silver NPs by polyol proses using fixed weight percentage of $\mathrm{AgNO}_{3}(2 \mathrm{mM})$ to dissolve it in fixed volume $(20 \mathrm{~mL})$ of Ethelene Glycol (EG) for making $\mathrm{AgNO}_{3}$ solution. Different weight percentage of Polyvinylpyrrolidone (PVP) solution was added as a stabilizer into the prepared solution of $\mathrm{AgNO}_{3}$ to make different molar concentrations. The prepared mixture was stirred for about 30 minutes at room temperature to make homogeneous solution. Finally, it was heated up to $\sim 70{ }^{0} \mathrm{C}$ to synthesize $\mathrm{Ag}$ NPs. It is to mention that silver nitrate $\left(\mathrm{AgNO}_{3}\right)$ and ethylene glycol (EG) were purchased from Merck (Emparta ${ }^{R}$ ) and Loba Chemie Pvt. Ltd, respectively. Polyvinylpyrrolidone (PVP-K30, average molecular weight of 40000) was purchased from SRL. For the present study, we have added different concentrations of PVP $(0.125 \mathrm{mM}-3 \mathrm{mM})$ keeping the concentration of $\mathrm{AgNO}_{3}$ fixed to a value of at $2 \mathrm{mM}$. This is to study the role of PVP concentration on the growth of Ag NPs.

The as-synthesized Ag NPs were characterized by ultraviolet-visible (UVVis) OA spectroscopy using a double-beam spectrophotometer (Jasco V-630) in the wavelength range of $340 \mathrm{~nm}$ to $700 \mathrm{~nm}$. For microstructural studies of the Ag NPs, we have used the X-ray diffractometer (PROTO AXRD). For $\mathrm{X}$-ray diffraction (XRD) measurements, all the samples were spin-coated on a glass substrates which were properly cleaned using a standard RCA cleaning method. XRD measurements were carried out using with $\mathrm{Cu} K_{\alpha}$ radiation $(\lambda$ $=0.154 \mathrm{~nm}$ ) and the intensity data were collected over a $2 \theta$ range of $20^{\circ}$ to $80^{\circ}$ with scanning steps of $0.02^{0}$. All the measurements were carried out at room temperature. Further to understand the role of PVP concentration on the growth of $\mathrm{Ag}$ NPs during the process of synthesis of we have carried out a systematic theoretical studies based on OA of Ag NPs using the effective 
medium theory [23,24]. The details of the theoretical studies including the methodology will be discussed later in this article.

\section{Experimental Results}

3.1 The dependence of particle size on the PVP concentration

To study the effects of surfactant (here PVP) on the size of the Ag NPs, XRD measurements were carried out on all the samples. Figure 1(a)shows the XRD patterns of the Ag NPs for different concentrations of PVP. For
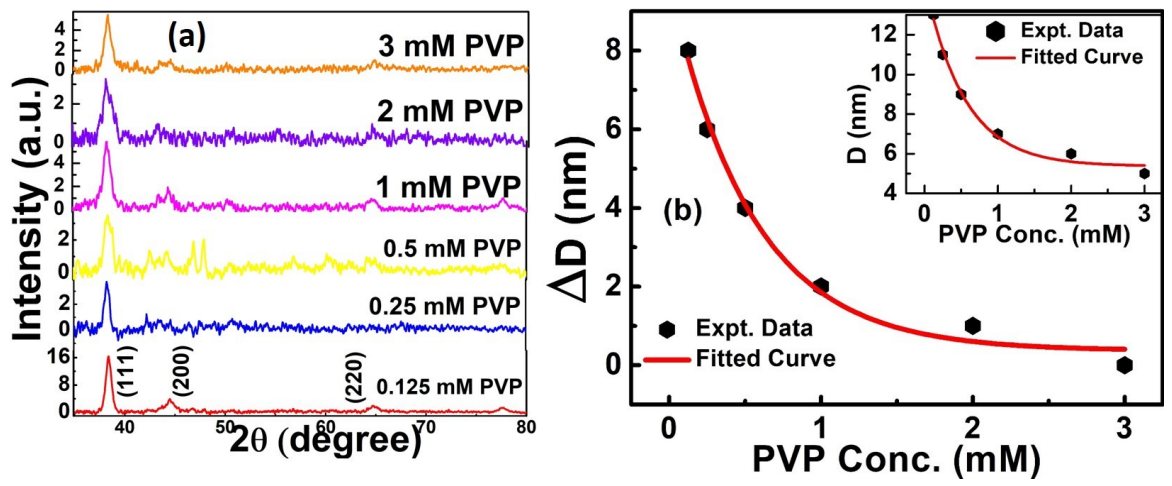

Fig. 1 (a) XRD patterns of Ag NPs synthesized for different PVP concentrations. (b) Variation of change $\Delta D$ with PVP concentration. Inset shows the variation of NP size D with PVP concentration

the lowest concentration of PVP $(0.125 \mathrm{mM})$, few XRD peaks are seen at $2 \theta$ positions of $38.4^{0}, 44.5^{0}, 64.7^{0}$, and $77.5^{0}$. These peaks are identified as the (111), (200), (220) and (311) crystal planes of face centered cubic (fcc) $\mathrm{Ag} 25$ - These are marked in the figure against each peak. With increase in PVP concentration, only (111) XRD peak retains indicating that the Ag particles preferably oriented towards the stable structure [26. From XRD data we did not observe any phase related to impurity present with the $\mathrm{Ag}$ in our samples. This observation clearly indicates that the synthesized Ag particles are of good quality. With a careful observation to the peak corresponding to (111) plane, one can find a systematic increase of the width with increase in PVP concentration. Such changes of the XRD peak width is indicative of reduction of crystallite size. For quantitative analysis, we have estimated the size of the crystallites, $D$, of Ag particles using Debye-Scherrer's equation

$$
D=\frac{K \lambda}{\beta \cos \theta},
$$

where $K=0.9, \lambda=1.54 \AA, \beta$ is FWHM of the XRD peak in radian, and $\theta$ is the XRD peak position. For the estimation, we have also taken into account 
the instrumental broadening $\beta_{0}=0.103$ to estimate the correct $\beta$ value using $\beta=\sqrt{\left(\beta_{m}\right)^{2}-\left(\beta_{0}\right)^{2}}$ where $\beta_{m}$ is the measured value of $\beta$. The size of the Ag NPs with different concentrations $(c)$ of the surfactant is estimated between $13 \mathrm{~nm}$ to $5 \mathrm{~nm}$ for the lowest to highest surfactant concentration $(0.125 \mathrm{mM}$ and $3 \mathrm{mM})$, respectively. The variation of change in particle size $(\Delta D)$ with the surfactant concentration $c$ is shown in figure 1(b) and inset shows the variation of NP size $(D)$ with PVP concentration $c$ considering $3 \mathrm{mM}$ precursor concentration as the reference. Initially, $\Delta D$ decreases very fast with increase in the value of $c$ and then decreases slowly showing a saturation behaviour. Such variation of $\Delta D$ with $c$ can be very well using an exponential decay function

$$
\Delta D=\Delta D_{0}+\Delta D_{m}\left[\exp \left(-\frac{c}{c_{0}}\right)\right]
$$

and from the fitting we have found the different fitting parameters as $\Delta D_{m}=$ $\sim 7.7 \mathrm{~nm}, \Delta D_{0} \sim 0.37 \mathrm{~nm}$, and $c_{0}=0.37 \mathrm{mM}$. Here $\Delta D_{m}$ is the maximum change in $\mathrm{Ag} \mathrm{NP}$ size for a change in $c$ from $0.125 \mathrm{mM}$ to $3 \mathrm{mM}$.

\subsection{Spectral shift of LSPR absorption for different PVP concentrations}

From the above discussions, it is evident that surfactant concentration plays an important role in the formation of Ag NPs and hence determining the size of the NPs. Here, we will study the effect of surfactant concentrations on the OA properties, especially LSPR properties of Ag NPs.Figure 2(a) shows the OA spectra of all the samples. A clear OA band centered at around $422 \mathrm{~nm}$
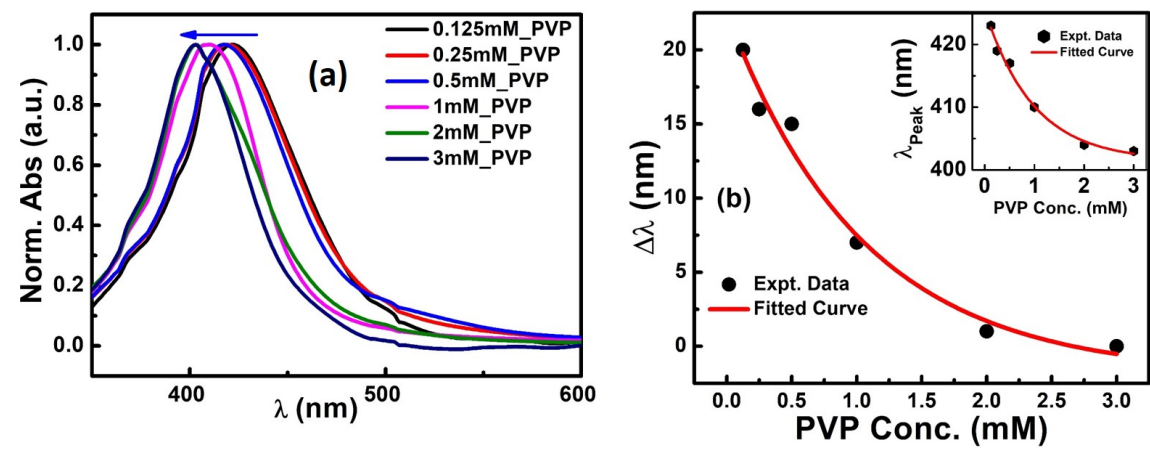

Fig. 2 (a) The change in LSPR peak position of Ag-NP solutions prepared with different molar concentrations of PVP. (b) The shift of LSPR peak position $(\Delta \lambda)$ with PVP concentration. $\Delta \lambda$ shows an exponential dependence with PVP concentration.Inset shows the peak position at different PVP concentration.

is observed for the PVP concentration of $0.125 \mathrm{mM}$. Appearance of such a peak in the OA spectrum with a sample containing of Ag NPs is due to the SPR absorption of the Ag NPs [27. Such LSPR bands also appear for other 
samples prepared for higher concentrations of PVP. A clear blueshift of the LSPR peak with increase in PVP concentration is seen. This blueshift is about $20 \mathrm{~nm}$ for the variation of PVP concentration from $0.125 \mathrm{mM}(422 \mathrm{~nm})$ to 3 $\mathrm{mM}$ (402 $\mathrm{nm}$. This appears to be a very large shift. Such blueshift of LSPR peak is indicative of reduction of Ag NP size 28 .

Figure 2(b) shows the observed variation of $\Delta \lambda$ with the different molar concentrations of the surfactant and inset shows the peak position at different PVP concentration. Here the values of $\Delta \lambda$ were calculated considering the data for $3 \mathrm{mM}$ as the reference. $\Delta \lambda$ is seen to decays exponentially with the concentration of PVP. This variation is well fitted using the following equation

$$
\Delta \lambda=\Delta \lambda_{0}+\Delta \lambda_{m}\left[\exp \left(-\frac{c}{c_{0}}\right)\right] .
$$

From the fitting we have found different important fitting parameters as $c_{0}=$ $0.96 \mathrm{mM}, \Delta \lambda_{0}=-1.9 \mathrm{~nm}$, and $\Delta \lambda_{m}=22 \mathrm{~nm}$ which is the maximum shift of LSPR peak position.

Eqn. (2) can be justified in a sense that the increasing surfactant concentration controls the NP's size which in turn affect the SPR which has a direct relationship with NP diameter. The critical concentration $c_{0}$ is actually correlated with the critical particle size above which there would not be any appreciable change in the LSPR peak and this will be discussed in later section. But the observed change in LSPR peak position cannot be explained only due to the variation of NP size as we should not ignore other crucial parameters like dielectric interface between the NP and the surrounding medium, and the role of polymer thickness which also controls the NP size. The surfactant actually covers the NP (core) as a shell which prevent the particles from further agglomeration. The core-shell ratio (in terms of radius or volume) plays a crucial role in the SPR which will be discussed in detail in the later section.

\section{Theoretical Model and Discussions}

UV-Vis absorption measurements of different Ag NP solutions with varying surfactant concentration $(c)$ show a noticeable LSPR peak shift $(\Delta \lambda)$ of $\sim 20$ $\mathrm{nm}$ [shown in figure 2(b)], which is found to be proportional to the variation of NP size, $(D)$. However, the NP size is not only controlled by PVP concentration but also depends on the surrounding dielectric medium [29] as well as the surfactant layer, which implies that the LSPR peak shift is not solely for the change in Size of the Ag NPs. In this section, we show that $\Delta \mathrm{D}$ is not sufficient enough to produce such a large $\Delta \lambda$ and SPR peak shift is also a result of the changing ratio between the radius of the NP (core) and the thickness of the surfactant layer (shell). For this purpose, we have considered the $\mathrm{Ag} \mathrm{NP}$ as the spherical core of radius, $R_{C}$, and the surfactant (PVP) layer surrounds the core as a spherical shell. Thus the synthesized Ag NPs were considered as core-shell structures. In our previous study [21, we have shown a systematic redshift of the SPR band with increase in concentration 
of precursor $\left(\mathrm{AgNO}_{3}\right)$ while keeping the surfactant concentration fixed. In this case, it was observed that average size of the Ag NPs were increased with increase in precursor concentration. This was confirmed through XRD and transmission electron microscopy (TEM) studies (average Ag NP sizes for different precursor concentrations measured from XRD and TEM studies were found to be consistent). It is to mention that with increase in NP size, the effective shell thickness of the surfactant (PVP) increases. Considering coreshell (Ag core PVP shell) structures we have explained the observed large redshift of the SPR band in this case. Similarly, for the present study also we have considered a core-shell structures of Ag NPs and PVP to account the observed large blueshift of the SPR band. This is shown schematically in figure 3. The core plus shell radius, $R_{S}$ [30], is schematically shown in Fig.3.

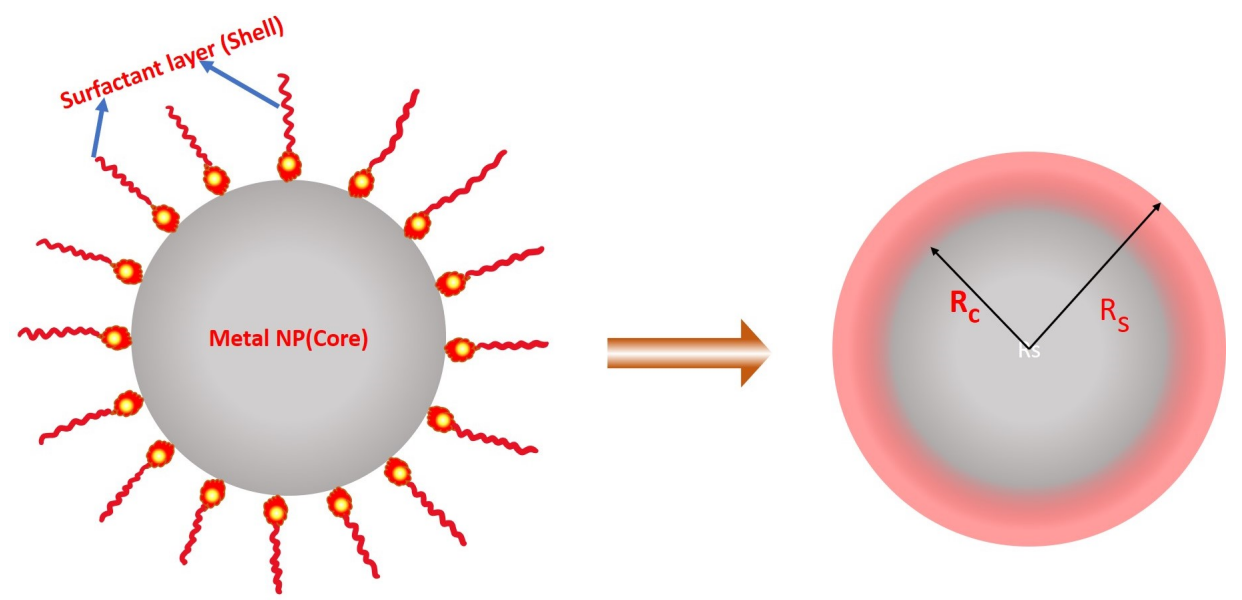

Fig. 3 Schematic diagram of Ag core and PVP shell nanostructure.

For the calculation of OA spectra for Ag NPs in PVP or EG (ethylene glycol) we have considered the effective medium theory for the electromagnetic response of the composite system 31. It is well known that the dielectric constant of the surrounding medium of the metal NPs plays an important role for the position of the SPR band 32. The equivalent dielectric constant of the core-shell [33] system can be expressed as

$$
\epsilon_{E}=f \epsilon_{C}+(1-f) \epsilon_{S},
$$

where $\epsilon_{E}$ is the effective dielectric constant of the composite system, $\epsilon_{C}$ is the dielectric constant of the core, $\epsilon_{S}$ is the dielectric constant of the shell, and $f$ is the volume fraction of the core with $f=\frac{R_{c}^{3}}{R_{S}^{3}}$. It will be shown that this $f$ plays a vital role to control the LSPR peak shift $(\Delta \lambda)$ depending up on the values of core radius and shell radius. 
To study the effects of Ag NP size in EG or PVP (without considering coreshell structure) on the OA spectra we have used the above mentioned effective medium theory modified by García et al 34. The details of the theoretical model can be found elsewhere [34,35]. This theory was successfully employed to calculate the $\mathrm{OA}$ data for $\mathrm{Au} 34,36, \mathrm{Ag} 37$, and $\mathrm{Al} 38$ NPs. Also this model was extended to calculate the OA spectra of alloys' metal NPs 39 , 40. For the present theoretical study, we have considered all the required parameters same as that in the case of [35] except the size (radius) of Ag NPs and the medium dielectric constant. The sizes of the (uncoated) Ag NPs were considered as estimated using XRD measurements and the medium dielectric constant was kept fixed to a value of 2.04. The optical constants of Ag were taken from the work of Johnson and Christy [41.

Figure 4(a) shows the theoretical normalized OA spectra of uncoated Ag NPs in EG for different sizes. The OA spectra corresponding to the Ag NP
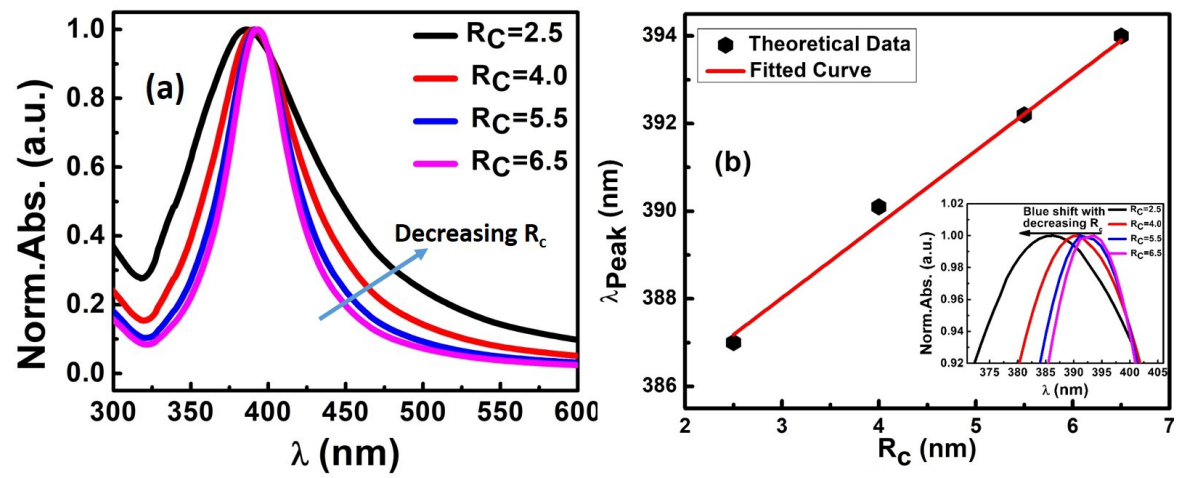

Fig. 4 (a) Theoretically calculated normalized OA spectra of uncoated Ag NPs in EG for different NP sizes. (b) Variation of OA peak position as a function of Ag NP radius. Inset shows the magnified SPR bands of (a).

radius of $2.5 \mathrm{~nm}$ shows an absorption band with a peak at around $387 \mathrm{~nm}$ and for the NP radius of $6.5 \mathrm{~nm}$, the OA peak appears at around $394 \mathrm{~nm}$. These absorption band corresponds to the surface plasmon resonance (SPR) of Ag NPs. With the decreasing NP size, a systematic shift of the absorption peak towards lower wavelength (blueshift) with the corresponding increase of its FWHM is observed. Such a peak shift with increased FWHM is due to the reduction of the Ag NPs size 35. Figure 4(b) shows the variation of the OA peak position as a function of $\mathrm{Ag} \mathrm{NP}$ radius and can be expressed as

$$
\lambda_{\text {Peak }}=\lambda_{0}+m\left[R_{c}\right],
$$

where, $\lambda_{0}=382 \mathrm{~nm}$, and the slope $m=1.83$.

The OA peak position changes linearly with a slope 1.83 towards a lower wavelength side with a decrease in NP. One can see that there is a peak shift 
of about $4 \mathrm{~nm}$ for the lowest sized to highest sized Ag NPs. Such an LSPR peak shift in OA spectra is due to the Ag NP size only [35]. This shift is much smaller than that we have observed experimentally $(\sim 20 \mathrm{~nm})$ in the present study. Thus the size of the Ag NPs alone is not sufficient to accommodate the experimentally observed LSPR peak shift in the OA spectra. Like our previous study, such large peak shift can be accommodated by considering the Ag-PVP core-shell structure. The process of synthesis likely to produce a capping layer of PVP on the Ag NPs [42]. Hence it is better to consider a coreshell nanostructure made of Ag PVP in EG. For the theoretical calculation of the OA spectra of such a system, we have extended the theoretical model of García et al 34 through Eqn. (4). It is to mention that the energy dependent dielectric constants of PVP were taken from the work of Ref. [43]. To obtain the LSPR peak positions as per the present experimental data, we have also varied the PVP shell thickness $\left(R_{S^{-}} R_{C}\right)$ through the parameter, $f$, via Eqn. (4) for each radius of Ag NPs measured using XRD technique [Figure 1].

Figure 5(a) shows the OA spectra of Ag-PVP core-shell NPs for different core-shell radius ( $R_{S}=R_{C}+\mathrm{PVP}$ shell thickness). It is to mention that for each case the core radius $\left(\mathrm{R}_{C}\right)$ was considered as it was for the uncoated $\mathrm{Ag}$ NPs [figure 4(a)]. The PVP shell thickness was chosen in such a way so that the theoretical OA spectra for each case would produce the OA band with LSPR peak positions as observed experimentally. Hence this process of choice will accommodate the required shift of LSPR peak position as seen experimentally (figure 2). One can see from figure 5(a) a clear blueshift of LSPR peak position with decrease in shell thickness. The variation of peak position as a function of $R_{S}$ is shown in figure $5(\mathrm{~b})$ and can be expressed as

$$
\lambda_{\text {Peak }}=\lambda_{0}+m\left[R_{s}\right],
$$

where, $\lambda_{0}=398 \mathrm{~nm}$, and the slope $m=1.79$. The peak position increases linearly with an increase of $R_{S}$. Consideration of core-shell nanostructures clearly ac-
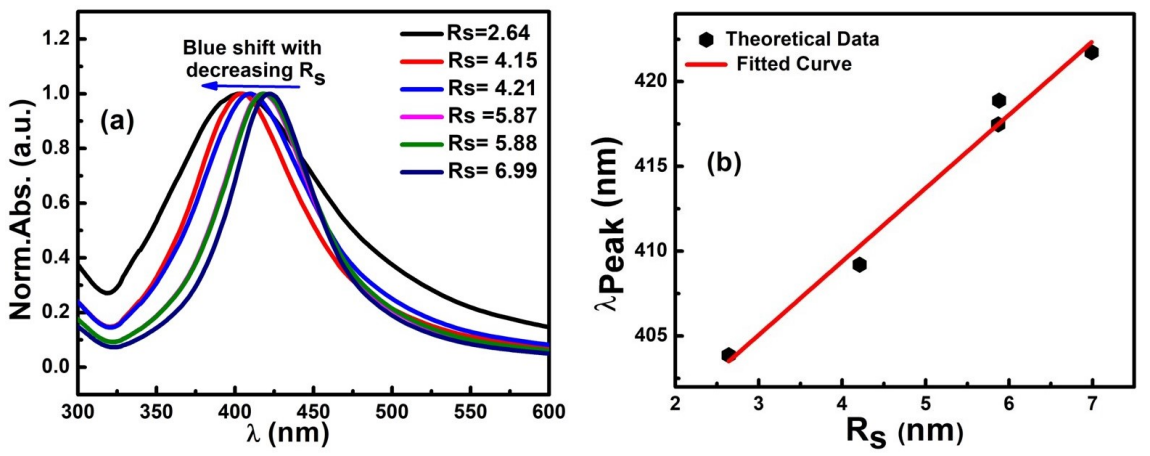

Fig. 5 (a) Theoretically calculated normalized OA spectra of AG-PVP core-shell nanostructures in EG for different sizes. (b) Variation of LSPR peak position as a function of radius of core-shell of Ag-PVP NPs. 
commodate the observed experimental LSPR peak positions of the OA spectra for different sized Ag NPs with varied PVP shell thickness. At this stage it is to important to understand the process of synthesis of Ag-PVP core-shell nanostructures and the role of PVP concentration for the control of both size of Ag NP as well as thickness of PVP shell. PVP shell plays a role of protective layer to control the the Ag NP particle size by reducing the inter-particle interactions. The metal NP surface and and the PVP layer have electrostatic interaction in nature and this interaction arises due to the charge transfer properties of PVP ligand with the metal NP surface. The increase in PVP layer thickness not only change the effective dielectric constant of the medium $\left(\epsilon_{E}\right)$ by changing $f$ or $R_{S}$ but also change the electrostatic interaction between the metal NP surface and the PVP layer by modifying the surface charge density of metal NP surface 22. Thus the modified surface charge density on metal NP surface and decrease in dielectric constant of the surrounding medium results in overall blue-shift of the LSPR peak.

\section{Conclusion}

We have observed the tunable optical response of PVP coated Ag NPs considering the varying particle size as well as shell thickness. LSPR of the Ag NPs having different surfactant concentrations shows a large blueshift $(\sim 20$ $\mathrm{nm}$ ) with increase in concentration of surfactant. From the XRD analysis of the samples, we found that the maximum change in particle size is $8 \mathrm{~nm}$ but from the theoretical model considering uncoated Ag NPs, it comes out that this change in NP size cannot produce the observed shift with changing core radius, $R_{C}$, up to $6.5 \mathrm{~nm}$. It is evident that the LSPR peak in the optical absorption spectra of Ag NPs has profound dependence on effective shell thickness, $R_{S}-R_{C}$, along with the particle size, $R_{C}$. We have found that the LSPR peak has a linear dependence on $R_{S}$. The finding of this work can also be useful to understand and analyze the optical response of other polymer coated plasmonic NPs.

\section{Acknowledgment}

Dilip Sao acknowledges Sumit Mukherjee and Aveek Banerjee for their valuable discussions.

\section{Ethical Approval}

This manuscript is compliance with scientific ethical standards and there are no other persons who satisfied the criteria for authorship but are not listed. We further confirm that the order of authors listed in the manuscript has been approved by all of us. 


\section{Consent to participate}

There is no involvement of animal. Consent to participate is not applicable for this manuscript.

\section{Consent to publish}

Informed consent was obtained from all authors who contributed in the study to publish this article.

\section{Author's Contribution}

All authors contributed to the study conception and design. Material preparation, data collection and analysis were performed by Dilip Sao, and Subhamay Pramanik. The first draft of the manuscript was written by Rajib Nath and Probodh K Kuiri and all authors commented on previous versions of the manuscript. All authors read and approved the final manuscript.

\section{Funding}

No funds, grants, or other support was received.

\section{Competing Interests}

All authors declare that they have no conflict of interest.

\section{Availability of data and materials}

The authors declare that all the data supporting the findings of this study are available within the article. The data that support the findings of this study are available on request from the corresponding author.

\section{References}

1. Maryamosadat Mavaei, Azam Chahardoli, Yalda Shokoohinia, Alireza Khoshroo, and Ali Fattahi, One-step Synthesized Silver Nanoparticles Using Isoimperatorin: Evaluation of Photocatalytic, and Electrochemical Activities, Scientific Reports, 10, 1762 (2020).

2. Jagdeep Singh,A. S. Dhaliwal, Plasmon-induced photocatalytic degradation of methylene blue dye using biosynthesized silver nanoparticles as photocatalyst, Environmental Technology, 41, 1520-1534 (2020).

3. B.Ajitha, Y. Ashok Kumar Reddy, P. Sreedhara Reddy, Hwan-Jin Jeon, and Chi Won Ahn, Role of capping agents in controlling silver nanoparticles size, antibacterial activity and potential application as optical hydrogen peroxide sensor, RSC Advances, 6, 3617136179 (2016). 
4. Jeevika, Alagan, and Dhesingh Ravi Shankaran, Functionalized silver nanoparticles probe for visual colorimetric sensing of mercury, Materials Research Bulletin, 83, 48-55 (2016).

5. Yan-Long Tai and Zhen-Guo Yang, Facile and Scalable Preparation of Solid Silver Nanoparticles $(<10 \mathrm{~nm})$ for Flexible Electronics, ACS Appl. Mater. Interfaces, 7,1710417111 (2015).

6. Hu, Daqiao, Jizhi Lin, Shan Jin, Yanlei Hu, Wenjun Wang, Rongrong Wang, and Bo Yang, Synthesis, structure and optical data storage properties of silver nanoparticles modified with azobenzene thiols, Materials Chemistry and Physics ,170,108-112 (2016).

7. Oliver Fenwick, Eduardo Coutino-Gonzalez, Didier Grandjean, Wouter Baekelant, Fanny Richard, Sara Bonacchi, Dirk De Vos, Peter Lievens, Maarten Roeffaers, Johan Hofkens Paolo Samori, Tuning the energetics and tailoring the optical properties of silver clusters confined in zeolites, Nature Materials ,15,1017-1022 (2016).

8. V. K. Sharma, R. A. Yngard, and Y. Lin, Silver nanoparticles: green synthesis and their antimicrobial activities, Advances in Colloid and Interface Science, 145, 83-96 (2009).

9. S. Mukherji and S. Agnihotri, Size-controlled silver nanoparticles synthesized over the range 5-100 $\mathrm{nm}$ using the same protocol and their antibacterial efficacy, RSC Advances, 4,3974-3983 (2014)

10. Lee, Sang Hun, and Bong-Hyun Jun, Silver Nanoparticles: Synthesis and Application for Nanomedicine ,International Journal of Molecular Sciences, 20, 865 (2019).

11. Xiaotong Liu, Dabing Li, Xiaojuan Sun, Zhiming Li, Hang Song, Hong Jiang Yiren Chen, Tunable Dipole Surface Plasmon Resonances of Silver Nanoparticles by Cladding Dielectric Layers, Scientific Reports,5,1-7 (2015).

12. Ihor Tokareva and Sergiy Minko, Tunable plasmonic nanostructures from noble metal nanoparticles and stimuli-responsive polymers, Soft Matter, 8, 5980-5987 (2012).

13. H. Baida, D. Christofilos, P. Maioli, A. Crut, N. Del Fatti, and F. VallÂtee, , Surface plasmon resonance spectroscopy of single surfactant-stabilized gold nanoparticles, Eur. Phys. J. D, 63, 293-299 (2011).

14. Nina Jiang,, Xiaolu Zhuo,and Jianfang Wang,Active Plasmonics: Principles, Structures, and Applications, Chem. Rev. ,118, 3054-3099 (2018).

15. D. Rithesh Raj, S. Prasanth, T.V. Vineeshkumar, C. Sudarsanakumar, Surface plasmon resonance based fiber optic dopamine sensor using green synthesized silver nanoparticles, Sensors and Actuators B: Chemical, 224,600-606 (2016).

16. P. Vasileva, B. Donkova, I. Karadjova, C. Dushkin, Synthesis of starch-stabilized silver nanoparticles and their application as a surface plasmon resonance-based sensor of hydrogen peroxide, Colloids and Surfaces A: Physicochemical and Engineering Aspects,382, 203-210 (2011).

17. Nilanjal Misra, Virendra Kumar, Lalit Borde, Lalit Varshney, Localized surface plasmon resonance-optical sensors based on radiolytically synthesized silver nanoparticles for estimation of uric acid, Sensors and Actuators B: Chemical, 178,371-378 (2013).

18. Christy L. Haynes, and Richard P. Van Duyne, Nanosphere lithography: a versatile nanofabrication tool for studies of size-dependent nanoparticle optics. J Phys Chem B, 105,5599-5611 (2001)

19. A. W.Powell, M. B. Wincott, A. A. R. Watt, H. E. Assender, and J. M. Smith, Controlling the optical scattering of plasmonic nanoparticles using a thin dielectric layer, J Appl Phys 113, 184311 (2013).

20. K.Khurana, N.Jaggi, Localized Surface Plasmonic Properties of Au and Ag Nanoparticles for Sensors: a Review,Plasmonics 16, 981-999 (2021).

21. Dilip Sao, Sandip Das, Subhamay Pramanik, Probodh K. Kuiri and Rajib Nath, Interplaying Role of Particle Size and Polymer Layer Thickness on the Large Tunable Optical Response of Polymer-coated Silver Nanostructures, Plasmonics,16,1319-1326 (2021).

22. Vikash Sharma, Chanderbhan Chotia, Tarachand, V. Ganesan and Gunadhor S. Okram, Influence of Particle Size and Dielectric Environment on Dispersion Behaviour and Surface Plasmon in Nickel Nanoparticles, Phys. Chem. Chem. Phys., 19, 14096-14106 (2017)

23. Tuck C Choy, Effective medium theory: principles and applications, 165, Oxford University Press (2015)

24. Yael Gutierrez, Dolores Ortiz, Rodrigo Alcaraz de la Osa, Jose M. Saiz, Francisco Gonzalez, and Fernando Moreno, Electromagnetic Effective Medium Modelling of Composites with Metal-Semiconductor Core-Shell Type Inclusions, Catalysts, 9, 626-639 (2019). 
25. Saini, Isha, Jyoti Rozra, Navneet Chandak, Sanjeev Aggarwal, Pawan K. Sharma, and Annu Sharma,Tailoring of electrical, optical and structural properties of PVA by addition of Ag nanoparticles, Materials Chemistry and Physics, 139, 802-810 (2013).

26. Salem, Salem S., Ehab F. El-Belely, Gniewko Niedbala, Maryam M. Alnoman, Saad ElDin Hassan, Ahmed Mohamed Eid, Tharwat I. Shaheen, Amr Elkelish, and Amr Fouda, Bactericidal and in-vitro cytotoxic efficacy of silver nanoparticles (Ag-NPs) fabricated by endophytic actinomycetes and their use as coating for the textile fabrics, Nanomaterials, 10, 2082 (2020).

27. Miguel A García, Surface plasmons in metallic nanoparticles: fundamentals and applications, Journal of Physics D: Applied Physics, 44, 283001-283020 (2011).

28. J Helmlinger, C Sengstock, C Groß-Heitfeld, C Mayer, T.A. Schildhauer, M Köller, M Epple, Silver nanoparticles with different size and shape: equal cytotoxicity, but different antibacterial effects, RSC advances, 6, 18490-18501 (2016).

29. O. Kylián, V. N. Popok, Applications of polymer films with gas-phase aggregated nanoparticles,Frontiers of Nanoscience, 15, 119-162 (2020).

30. Vadim A Markel, Introduction to the Maxwell Garnett approximation: tutorial,JOSA A,33,1244-1256 (2016)

31. JC Maxwell Garnett, Colours in metal glasses and in metallic films,Philosophical Transactions of the Royal Society of London. Series A, Containing Papers of a Mathematical or Physical Character, 203,385-420 (1904).

32. Jayanta K Majhi , Probodh K Kuiri, Enhancement of spectral shift of plasmon resonances in bimetallic noble metal nanoparticles in core-shell structure,Journal of Nanoparticle Research, 22, 1-12 (2020).

33. Anton Kuzma, Martin Weis, Sona Flickyngerova, Jan Jakabovic, Alexander Satka, Edmund Dobrocka, Juraj Chlpik, Julius Cirak, Martin Donoval, and Peter Telek, Influence of surface oxidation on plasmon resonance in monolayer of gold and silver nanoparticles, Journal of Applied Physics, 112, 103531-103535 (2012).

34. M.A.Garciía, J.Llopis, and Paje S. E., A simple model for evaluating the optical absorption spectrum from small Au-colloids in sol gel films, Chemical Physics Letters ,315,313320 (1999)

35. Jayanta K Majhi, Atis C Mandal, and Probodh K Kuiri, Theoretical Calculation of Optical Absorption of Noble Metal Nanoparticles Using a Simple Model: Effects of Particle Size and Dielectric Function, Journal of Computational and Theoretical Nanoscience, 12, 2997-3005 (2015).

36. P K Kuiri and D. P. Mahapatra, Surface Plasmon Resonance in Ag Nanoparticles Synthesized in Silica Glass by Low-Energy High-Fluence Ion Implantation,Advanced Science, Engineering and Medicine,6,290-295 (2014).

37. P K Kuiri, Size saturation in low energy ion beam synthesized nanoparticles in silica glass: $50 \mathrm{keV} \mathrm{Ag-ions} \mathrm{implantation,} \mathrm{a} \mathrm{case} \mathrm{study,} \mathrm{Journal} \mathrm{of} \mathrm{Applied} \mathrm{Physics,} \mathrm{108,} \mathrm{054301-}$ 054306 (2010)

38. P K Kuiri, Control of Ultraviolet Surface Plasmon Absorption of Al Nanoparticles by Changing Particle Size, Shape, Interaction, and Medium Dielectric Constant, Plasmonics , 15,933-940 (2020).

39. Jayanta K Majhi and P K Kuiri, Spectral Tuning of Plasmon Resonances of Bimetallic Noble Metal Alloy Nanoparticles Through Compositional Changes,Plasmonics, 15,797-804 (2019).

40. P K Kuiri, Tailoring localized surface plasmons in Ag-Al alloys nanoparticles, Journal of Alloys and Compounds, 826, 154250-154254 (2020).

41. Peter B Johnson and R-W J Christy, Optical constants of the noble metals, Physical review B,6,4370-4379 (1972).

42. Ali Mirzaei, Kamal Janghorban, Babak Hashemi, Maryam Bonyani, Salvatore Gianluca Leonardi, and Giovanni Neri, Characterization and optical studies of PVP-capped silver nanoparticles, Journal of Nanostructure in Chemistry 7, 37-46 (2017)

43. T. A. F. Konig, P. A. Ledin, J. S Kerszuli, M. A. Mahmoud, M. A. El-Sayed, J. R. Reynolds, and V. V. Tsukruk, Electrically Tunable Plasmonic Behavior of Nanocube Polymer Nanomaterials Induced by a Redox Active Electrochromic Polymer, ACS nano, 8, 6182-6192 (2014). 